\title{
Predictors Associated with Survival Among Elderly In-Patients Who Receive Cardiopulmonary Resuscitation in Japan: An Observational Cohort Study
}

\author{
Tetsuro Hayashi, MD, MPH ${ }^{1,2}$, Masato Matsushima, MD, MPH, PhD², Seiji Bito, MD, MSHS , \\ Natsuko Kanazawa, RPT, MPH ${ }^{3,4}$, Norihiko Inoue, $\mathrm{MD}^{3,4}$, Sarah Kyuragi Luthe, MD, MPH ${ }^{5}$, and \\ Christina C. Wee, MD, MPH'
}

\begin{abstract}
'Division of Clinical Epidemiology, National Hospital Organization Tokyo Medical Center, Tokyo, Japan; ${ }^{2}$ Division of Clinical Epidemiology, The Jikei University School of Medicine, Tokyo, Japan; ${ }^{3}$ Department of Clinical Data Management and Research, Clinical Research Center, National Hospital Organization Headquarters, Tokyo, Japan; ${ }^{4}$ Department of Health Policy and Informatics, Tokyo Medical and Dental University Graduate School, Tokyo, Japan; 5 Department of Anesthesiology and Critical Care Medicine, Asahikawa Medical University, Asahikawa, Hokkaido, Japan; ${ }^{6} \mathrm{General}$ Medicine and Primary Care, Department of Medicine, Beth Israel Deaconess Medical Center, Boston, MA, USA.
\end{abstract}

BACKGROUND: Little is known about the outcomes of in-hospital cardiopulmonary resuscitation (CPR) in Asian populations including elderly patients in Japan. OBJECTIVE: To determine the survival outcome of inhospital CPR among elderly patients in Japan, and to identify predictors associated with survival.

DESIGN: Retrospective cohort study in 81 Japanese hospitals from April 1, 2010 to March 31, 2016.

PATIENTS: We included elderly patients (age $\geq 65$ years) who received CPR after 2 days of hospitalization.

MAIN MEASURES: The primary outcome was survival at hospital discharge and the secondary outcomes were the discharge disposition and consciousness level of patients who survived to hospital discharge. To determine predictors associated with survival after in-hospital CPR, we fit multivariable models for patient-level and institutionallevel factors.

KEY RESULTS: Among the 5365 patients who received CPR, 595 (11\%) survived to discharge. Of those who survived to discharge, $46 \%$ of patients were discharged home, and $10 \%$ of patients were comatose at discharge. Older age and higher burden of comorbidities were associated with reduced survival. The adjusted OR was 0.35 (95\% CI, 0.22-0.55) for age $\geq$ 90 years compared to age 65-69 years, and 0.68 (95\% CI, 0.48-0.97) for Charlson Comorbidity Index score of $\geq 4$ compared with score of 0 . Other predictors of reduced survival included receiving $\mathrm{CPR}$ on weekends compared to weekdays (AOR, 0.63; 95\% CI, 0.51-0.77) and in small hospitals compared to large hospitals (AOR, 0.58; 95\% CI, 0.40-0.83).

$\overline{\text { Presentation }}$

We presented an earlier version of the manuscript at the 2018 SGIM annual meeting on April 12, 2018.

Electronic supplementary material The online version of this article (https://doi.org/10.1007/s11606-018-4747-5) contains supplementary material, which is available to authorized users.

Received April 13, 2018

Revised October 9, 2018

Accepted November 1, 2018

Published online November 27, 2018
CONCLUSIONS: Among elderly patients in Japan, the survival rate of in-hospital CPR was approximately one in ten, and less than half of these patients were discharged home. In addition to older age and higher illness burden, receiving CPR on weekends and/or in small hospitals were significant predictors of reduced survival. These findings should be considered in advanced care planning discussions with elderly patients to avoid subjecting patients to CPR that are likely futile.

KEY WORDS: aging; end-of-life care; decision making; evidence base medicine.

J Gen Intern Med 34(2):206-10

DOI: $10.1007 / \mathrm{s} 11606-018-4747-5$

(c) Society of General Internal Medicine 2018

\section{INTRODUCTION}

Whether patients should receive cardiopulmonary resuscitation (CPR) is an important decision encountered by elderly patients and clinical teams; however, outcome data on inpatient CPR is unclear. Additionally, it has been reported that elderly patients and physicians overestimate the chance of survival after CPR when deciding whether a do-notresuscitate (DNR) order might be appropriate. ${ }^{1-5}$ Therefore, providing accurate outcomes on CPR may influence the decision-making on CPR for hospitalized elderly patients. ${ }^{6}$

A recent systematic review including 29 studies showed that the survival rate of CPR among elderly inpatients ranges from 11.6 to $18.7 \%$ and declines with increasing age. The vast majority of these patients were from the USA, ${ }^{7}$ and survival rate after CPR has also been shown to vary by race. ${ }^{8} \mathrm{~A}$ previous study using Medicare data reported that black and other non-white patients had a higher likelihood of receiving in-hospital CPR but had lower odds of survival. ${ }^{9}$

In contrast, only a few studies have examined the outcomes of in-hospital CPR in Asian populations including elderly patients in Japan, who have the longest life expectancy in the world. ${ }^{10}$ In Japan, more than $70 \%$ of hospitalized patients are 
aged $\geq 65$ years, approximately three in four die in the hospital, and two in ten do not have DNR orders. ${ }^{11}$ In this context, we used a large population-based database from 81 Japanese hospitals to investigate the survival rate after CPR among hospitalized elderly patients and to identify predictors associated with survival.

\section{METHODS}

\section{Date Source}

Patients' data were extracted from the Diagnosis Procedure Combination (DPC) data ${ }^{12}$ of the National Hospital Organization (NHO) Network in Japan. The DPC is nationally used for health care insurance claims for health service rendered in acute care hospitals in Japan, similar to medical claim codes used in the USA. ${ }^{13,14}$ The NHO is the largest hospital network in Japan, and stores DPC data from 81 acute care hospitals, typically teaching/tertiary hospitals in each district, affiliated with the NHO for administration and clinical information analysis. The DPC data includes hospital administrative data and discharge abstracts: unique identifiers of hospitals; characteristics of patients; admission and discharge status; diagnoses and comorbidities at admission, and complications after admission recorded in the International Classification of Diseases, Tenth Revision (ICD-10); surgical and non-surgical procedures; drugs and devices used; and length of stay. ${ }^{13}$ To optimize the accuracy of medical information, attending physicians are responsible for registering the diagnoses. This retrospective study was approved by the Institutional Ethics Committees at the National Hospital Organization and Tokyo Medical Center.

Study Sample. This study included patients aged $\geq 65$ years who were hospitalized and received CPR 2 days after admission from April 1, 2010 to March 31, 2016. We excluded patients who received CPR within 2 days of hospitalization due to the inability to distinguish patients who were in cardiac arrest on arrival to the hospitals. The procedure of CPR was identified as "Closed-chest cardiac massage (J046)" from the DPC database. For patients who went into more than one cardiac arrest event and received multiple CPR episodes during their hospitalization, we only included the first CPR in our analysis.

Outcome Variables. Our primary outcome was survival to hospital discharge among elderly in-patients who received CPR after the second day of their hospitalization. As secondary outcomes, we investigated the discharge disposition (home, nursing facilities, or other hospitals) of patients who survived to hospital discharge and the patient's level of consciousness at discharge (comatose or not). Consciousness level was categorized based on the Japan Coma Scale where comatose is defined by the three-digit code of 100,200 , or $300 .{ }^{15}$
Potential Predictors. We considered the following potential patient-level and institutional-level predictors: patient sex, age, body mass index (BMI), consciousness level on admission (based on the Japan Coma Scale), ${ }^{15}$ admitting diagnosis, admitting comorbidities (based on the Charlson comorbidity index) ${ }^{16}$ hospital size (defined by number of hospital beds: < 300, small; 300-499, medium; and $\geq 500$, large), days from admission to CPR, and day of week of CPR (weekend vs. weekday). We also considered whether patients received the following interventions within 3 days prior to CPR in order to assess pre-resuscitation interventions: intensive care unit (ICU) admission, enteral nutrition, total parenteral nutrition, vasopressor use, and mechanical ventilation (defined by noninvasive positive pressure ventilation and/or invasive mechanical ventilation).

Statistical Methods. We compared baseline patient characteristics using chi-square test. To determine the predictors associated with survival after in-hospital CPR at the level of alpha $=0.05$, we fit unadjusted and adjusted logistic regression models for each response using a forward selection approach. Non-significant variables were added sequentially; no potential confounders that altered estimates of significant factors by more than $10 \%$ were identified. Thus, candidate explanatory variables associated with survival were identified. Next, we analyzed the candidate variables using generalized estimating equations with a logit link function where the clustering effect associated with hospitals is accounted for by the robust sandwich standard error estimator. We tested the significance of BMI in a subset of patients with complete data as BMI was missing in 885 patients (16\%), and found no significant importance. We also performed a sensitivity analysis excluding patients who underwent multiple CPR events. All analyses were performed with STATA 12 software (STATA Corp, College Station, TX). All $P$ values were twotailed and considered statistically significant with $P<0.05$.

\section{RESULTS}

Patients. We identified $1,478,934$ patients aged $\geq 65$ years who were hospitalized for more than 2 days in 81 Japanese hospitals from April 1, 2010 to March 31, 2016. Among these, 78,360 patients experienced cardiac arrest after 2 days of admission, and 5365 patients received CPR.

Descriptive Statistics. Among the 5365 patients who received CPR, 595 patients (11\%) survived to hospital discharge. Of the 4770 patients who died after CPR, 3768 patients (79\%) died within the same day of receiving CPR. Overall, the median length of stay was 22 days, and the median length from hospital admission to event of cardiac arrest was 16 days. Table 1 shows the characteristics of the patients who 
Table 1 Characteristics of Patients Who Received In-Hospital Cardiopulmonary Resuscitation $(N=5365)$

\begin{tabular}{|c|c|c|c|c|}
\hline \multirow[t]{2}{*}{ Characteristics } & \multicolumn{2}{|c|}{ Sample } & \multirow{2}{*}{$\begin{array}{l}\text { Survival } \\
\text { rate }\end{array}$} & \multirow{2}{*}{$\begin{array}{l}P \\
\text { value* }\end{array}$} \\
\hline & $\mathbf{n}$ & $(\%)$ & & \\
\hline Overall & 5365 & $(100.0)$ & 11.1 & \\
\hline \\
\hline Male & 3375 & $(62.9)$ & 10.8 & 0.35 \\
\hline Female & 1990 & $(37.1)$ & 11.6 & \\
\hline \multicolumn{5}{|l|}{ Age } \\
\hline $65-69$ & 677 & $(12.6)$ & 13.6 & 0.006 \\
\hline $70-74$ & 890 & (16.6) & 9.9 & \\
\hline $75-79$ & 1181 & $(22.0)$ & 12.1 & \\
\hline $80-84$ & 1308 & $(24.4)$ & 11.1 & \\
\hline $85-89$ & 888 & (16.6) & 11.3 & \\
\hline$\geq 90$ & 421 & $(7.9)$ & 6.4 & \\
\hline \multicolumn{5}{|l|}{ Body Mass Index ${ }^{a}$} \\
\hline$<18.5$ & 1251 & $(23.3)$ & 9.4 & 0.06 \\
\hline $18.5-25$ & 3487 & $(65.0)$ & 11.4 & \\
\hline$\geq 25$ & 627 & (11.7) & 12.8 & \\
\hline Comatose on admission & 296 & $(5.5)$ & 15.2 & 0.02 \\
\hline \multicolumn{5}{|l|}{ Admitting diagnosis } \\
\hline Cancer & 984 & $(18.3)$ & 6.5 & $<0.001$ \\
\hline Pneumonia & 543 & $(10.1)$ & 9.8 & \\
\hline Congestive heart failure & 542 & (10.1) & 13.5 & \\
\hline $\begin{array}{l}\text { Cerebrovascular } \\
\text { diseases }\end{array}$ & 377 & $(7.0)$ & 13.0 & \\
\hline $\begin{array}{l}\text { Other respiratory } \\
\text { diseases }\end{array}$ & 316 & $(5.9)$ & 7.9 & \\
\hline Traumatic diseases & 291 & $(5.4)$ & 14.8 & \\
\hline Ischemic heart diseases & 290 & $(5.4)$ & 21.7 & \\
\hline $\begin{array}{l}\text { Gastroenterological } \\
\text { diseases }\end{array}$ & 183 & (3.4) & 12.0 & \\
\hline $\begin{array}{l}\text { Other infectious } \\
\text { diseases }\end{array}$ & 168 & $(3.1)$ & 4.8 & \\
\hline Aortic dissection & 122 & $(2.3)$ & 13.1 & \\
\hline Neurological diseases & 101 & (1.9) & 13.9 & \\
\hline Renal failure & 98 & (1.8) & 12.2 & \\
\hline Hematological diseases & 92 & (1.7) & 1.1 & \\
\hline Arrhythmia & 88 & (1.6) & 33.0 & \\
\hline Other & 1170 & $(21.8)$ & 10.5 & \\
\hline \multicolumn{5}{|c|}{ Charlson Comorbidity Index } \\
\hline 0 & 1669 & $(31.1)$ & 12.2 & 0.009 \\
\hline 1 & 1415 & $(26.4)$ & 12.4 & \\
\hline 2 & 1016 & (18.9) & 10.5 & \\
\hline 3 & 714 & (13.3) & 8.8 & \\
\hline \multirow{2}{*}{\multicolumn{5}{|c|}{ Pre-resuscitation interventions }} \\
\hline & & & & \\
\hline $\begin{array}{l}\text { Intensive care unit } \\
\text { admission }\end{array}$ & 532 & $(9.9)$ & 17.9 & $<0.001$ \\
\hline Enteral nutrition & 820 & $(15.3)$ & 11.2 & 0.90 \\
\hline Total parental nutrition & 758 & (14.1) & 8.8 & 0.03 \\
\hline Vasopressor use & 1103 & $(20.6)$ & 12.1 & 0.21 \\
\hline Mechanical ventilation & 854 & (15.9) & 13.3 & 0.02 \\
\hline \multicolumn{5}{|c|}{ Days from admission to CPR } \\
\hline $3-7$ & 1383 & $(25.8)$ & 14.3 & $<0.001$ \\
\hline $8-14$ & 1064 & (19.8) & 12.3 & \\
\hline $15-28$ & 1174 & (21.9) & 9.5 & \\
\hline$\geq 29$ & 1744 & $(32.5)$ & 8.8 & \\
\hline \multicolumn{5}{|l|}{ Day of CPR } \\
\hline Weekday & 3695 & $(68.9)$ & 12.4 & $<0.001$ \\
\hline Weekend & 1670 & $(31.1)$ & 8.3 & \\
\hline \multicolumn{5}{|c|}{ Hospital size (number of hospital beds) } \\
\hline$<300$ & 845 & $(15.8)$ & 6.2 & $<0.001$ \\
\hline $300-499$ & 3094 & (57.7) & 12.0 & \\
\hline$\geq 500$ & 1426 & (26.6) & 12.1 & \\
\hline
\end{tabular}

Abbreviation: CPR cardiopulmonary resuscitation

*All the comparisons were made using chi-square tests

${ }^{a} 16 \%$ missing

${ }^{b}$ Including sepsis

received CPR and the survival rates. The median age was 79 years (IQR, 73-84 years) and 63\% were male. Of 231 patients who underwent multiple CPR events during hospitalization, 32 (14\%) patients survived to hospital discharge.

Status Among Patients Who Survived to Discharge After CPR. Among 595 patients who survived to discharge, the median length of hospitalization from receiving CPR to discharge was 46 days, and $59(10 \%)$ patients were comatose at discharge (Table 2). Of 581 patients with data available on discharge dispositions, $279(48 \%)$ patients were transferred to other hospitals, 266 (46\%) patients were discharged home, and 18 (3\%) patients were transferred to nursing facilities.

Multivariable Analysis. Table 3 presents significant predictors associated with survival to hospital discharge after adjustment. Age and higher burden of comorbidities were significant predictors of reduced survival. Admitting diagnoses of cancer, infectious disease, and hematological disease were also significant predictors of reduced survival, while admitting diagnoses of ischemic heart disease, arrhythmia on admission, and ICU admission prior to resuscitation were associated with greater survival. The patients who received CPR after 2 weeks from admission were less likely to survive. Receiving CPR on weekends and/or in small hospitals were also significant predictors of reduced survival. There was no effect modification between hospital size and receiving CPR on weekends or between hospital size and ICU admission. Other factors such as sex, BMI, consciousness level on admission, and pre-resuscitation interventions of enteral nutrition, total parental nutrition, vasopressor treatment, and mechanical ventilation were not significant predictors or confounders. In the sensitivity analysis excluding patients who underwent multiple CPR events, the significance and direction of the effect of the predictors were not different from the result in the main analysis.

\section{DISCUSSION}

In our study of over 5000 elderly patients who received inhospital CPR in Japan, approximately one in ten survived, and less than half of these patients were discharged home. In

Table 2 Status Among Patients Who Survived to Discharge After Cardiopulmonary Resuscitation $(N=595)$

\begin{tabular}{lll}
\hline \hline & $\boldsymbol{n}$ & $\mathbf{( \% )}$ \\
\hline Discharge disposition $^{\mathrm{a}}$ & & \\
Other hospitals & 279 & $(48.0)$ \\
Home & 266 & $(45.8)$ \\
Nursing facilities & 18 & $(3.1)$ \\
Other & 18 & $(3.1)$ \\
Neurological status & 59 & $(9.9)$ \\
Comatose at discharge & & \\
\hline
\end{tabular}

a581 patients' data (98\%) were available on discharge dispositions 
Table 3 Multivariable Analyses for Predictors Associated with Survival to Hospital Discharge (adjusted for the year of admission)

\begin{tabular}{|c|c|}
\hline Predictors & Odds ratio $(95 \% \mathrm{CI})$ \\
\hline \multicolumn{2}{|l|}{ Age } \\
\hline $65-69$ & Reference \\
\hline $70-74$ & $0.69(0.50-0.95)$ \\
\hline $75-79$ & $0.84(0.63-1.12)$ \\
\hline $80-84$ & $0.70(0.52-0.94)$ \\
\hline $85-89$ & $0.71(0.52-0.97)$ \\
\hline$\geq 90$ & $0.35(0.22-0.55)$ \\
\hline \multicolumn{2}{|l|}{$\bar{A} d m i t t i n g ~ d i a g n o s i s$} \\
\hline Pneumonia & Reference \\
\hline Cancer & $0.61(0.41-0.90)$ \\
\hline Congestive heart failure & $1.32(0.90-1.94)$ \\
\hline Cerebrovascular diseases & $1.16(0.76-1.76)$ \\
\hline Other respiratory diseases & $0.73(0.44-1.21)$ \\
\hline Traumatic diseases & $1.46(0.94-2.26)$ \\
\hline Ischemic heart diseases & $1.84(1.21-2.80)$ \\
\hline Gastroenterological diseases & $1.19(0.69-2.03)$ \\
\hline Other infectious diseases ${ }^{\mathrm{a}}$ & $0.40(0.19-0.87)$ \\
\hline Aortic dissection & $1.04(0.56-1.92)$ \\
\hline Neurological diseases & $1.36(0.72-2.59)$ \\
\hline Renal failure & $1.14(0.58-2.25)$ \\
\hline Hematological diseases & $0.08(0.01-0.63)$ \\
\hline Arrhythmia & $3.96(2.30-6.79)$ \\
\hline Other & $1.02(0.72-1.44)$ \\
\hline \multicolumn{2}{|l|}{ Charlson Comorbidity Index } \\
\hline 0 & Reference \\
\hline 1 & $0.99(0.79-1.24)$ \\
\hline 2 & $0.83(0.64-1.07)$ \\
\hline 3 & $0.75(0.55-1.03)$ \\
\hline$\geq 4$ & $0.68(0.48-0.97)$ \\
\hline \multicolumn{2}{|l|}{ Pre-resuscitation intervention } \\
\hline Intensive care unit admission & $1.38(1.06-1.80)$ \\
\hline \multicolumn{2}{|l|}{ Days from admission to CPR } \\
\hline $3-7$ & Reference \\
\hline $8-14$ & $0.88(0.69-1.12)$ \\
\hline $15-28$ & $0.68(0.53-0.88)$ \\
\hline$\geq 29$ & $0.67(0.53-0.85)$ \\
\hline \multicolumn{2}{|l|}{ Day of CPR } \\
\hline Weekday & Reference \\
\hline Weekend & $0.63(0.51-0.77)$ \\
\hline \multicolumn{2}{|l|}{ Number of hospital beds } \\
\hline$<300$ & $0.58(0.40-0.83)$ \\
\hline $300-499$ & $1.05(0.83-1.32)$ \\
\hline$\geq 500$ & Reference \\
\hline
\end{tabular}

The ORs of the predictors associated with survival or reduced survival after in-hospital CPR at the level of alpha $=0.05$ are presented in bold Abbreviation: CPR cardiopulmonary resuscitation

${ }^{a}$ Including sepsis

addition to older age and higher illness burden, receiving CPR on weekends and/or in small hospitals were significant predictors of reduced survival; in contrast, ischemic heart disease, arrhythmia on admission, and ICU admission before resuscitation were associated with greater survival.

To the best of our knowledge, this is the largest study outside of the USA examining the outcomes of in-hospital CPR among elderly patients, and the first study focusing on an Asian population. Further, this study considered preresuscitation interventions and institutional-level factors in addition to baseline characteristics on hospital admission.

Approximately half of the patients who survived to discharge after CPR were discharged home. In contrast, another half of those were transferred to other hospitals; one in ten were comatose at discharge. Many of the patients transferred to other hospitals were likely to move to rehabilitation hospitals or long-term care hospitals rather than other acute care hospitals though these data were not available in our study. Nonetheless, these findings suggest that these patients had physical complications after CPR and required longer-term medical treatment.

Our findings are consistent with prior data reporting the association between older age and reduced survival. ${ }^{7,} 9 \mathrm{~A}$ systematic review showed that the pooled survival rate after CPR was $18.7 \%$ for patients between 70 and 79 years old, $15.4 \%$ for those between 80 and 89 years old, and $11.6 \%$ for 90 years and older. ${ }^{7}$ The survival rates of in-hospital CPR in the present study were even lower than that of prior studies. This difference may be partly explained by the different inclusion criteria of when CPR was performed; for example, we excluded patients who received CPR within 2 days of hospitalization due to our inability to distinguish patients who were in cardiac arrest on arrival to the hospitals.

Our finding that arrhythmia, ischemic heart disease, and ICU admission were associated with increased survival rates likely reflects increased use of cardiac monitoring and rapid response to event. ${ }^{17}$ In addition, patients with arrhythmia or ischemic heart disease might be more likely to have initial rhythms of ventricular fibrillation or ventricular tachycardia, which are more responsive to CPR. ${ }^{18,}{ }^{19}$ Furthermore, there may be a selection bias in which patients are admitted to ICU based on physician's perception of patient's likelihood to benefit from ICU care. In contrast, cancer, infection, hematological disease, and higher burden of comorbidities were independently predictive of in-hospital mortality, and these findings are in line with previous studies. ${ }^{9,} 17$ These predictors of poor prognosis can be applied to advanced care planning discussions and guide decision-making about do-notresuscitate orders.

Our finding that receiving CPR on weekends compared to weekdays as an independent predictor of reduced survival is consistent with a previous study demonstrating that patients who received CPR during day on weekdays were more likely to survive, compared to those who received CPR during day on weekends. ${ }^{20}$ Our findings also indicate that receiving CPR in smaller hospitals compared to larger hospitals were associated with reduced survival. The underlying mechanism of reduced survival after CPR on weekends and in small hospitals may be explained by different hospital staffing patterns in these settings. The difference in the availability of rapid response systems (RRS) between larger hospitals and smaller hospitals may be another explanation, since the shortage of medical staff is considered to be the main barrier of implementing RRS. ${ }^{21}$ Addressing institutional systems, such as implementing RRS among small hospitals during weekends, may improve important outcomes associated with CPR.

While our study leverages a rich dataset, it is not without limitations. First, our definition of CPR was based on the DPC data in Japan, and the definition of CPR has not been validated in this data, although a previous validation study on the DPC data shows that the sensitivity and specificity of common procedures exceeds $90 \%$ and the sensitivity and specificity 
of primary diagnoses were $79 \%$ and $93 \% .^{22}$ Second, our analyses were limited to patients who received CPR after 2 days of hospitalization, which may have underestimated the survival rate in our study. Third, the dataset had limited information on other potential predictors of survival such as initial arrest rhythm, presence of witness, use of telemetry, and the availability of rapid response systems. In addition, the dataset had also limited information on other outcomes such as physical function, quality of life, and details of hospitals to which patients were transferred.

In conclusion, among elderly patients in Japan, the survival rate of in-hospital CPR after 2 days of hospitalization was approximately one in ten, and less than half of these patients were discharged home. In addition to older patient age and higher illness burden, receiving CPR on weekends and in small hospitals were significant predictors of reduced survival. These findings suggest that patients' baseline status should be considered in advanced care planning discussions with elderly patients to avoid subjecting patients to CPR that are likely futile. Moreover, future studies should identify potential system factors that might underlie differences in outcomes after CPR between large and small hospitals.

Acknowledgments: We thank Mr. Masaya Nakadera for the assistance with data extraction and thank Dr. Suminobu Ito and Dr Hiromasa Horiguchi for organizing this research.

Corresponding Author: Tetsuro Hayashi, MD, MPH; Division of Clinical Epidemiology National Hospital Organization Tokyo Medical Center, Tokyo, Japan (e-mail: thayashi@ntmc-hosp.jp).

Funding This study was supported by a research grant for postgraduate students from Jikei University School of Medicine. The study sponsor had no role in the study design, data collection, analysis and interpretation, report writing, or the decision to submit the article for publication. MM is the program director of Jikei Clinical Research Program for Primary Care, and TH was a former trainee of the Jikei Clinical Research Program for Primary Care. When this study was conducted, TH was the Shigeaki Hinohara, MD, International Primary Care Fellow at Beth Israel Deaconess Medical Center (BIDMC) which was supported by the Ryoichi Sasakawa Fellowship Fund at BIDMC. SL was supported by grants from World Bank and Kamiyama Foundation. $C W$ is supported by Midcareer Mentorship Award from the National Institutes of Health (K24DK087932).

\section{Compliance with Ethical Standards:}

Conflict of Interest: The authors declare that they do not have a conflict of interest.

\section{REFERENCES}

1. Miller DL, Jahnigen DW, Gorbien MJ, Simbartl L. Cardiopulmonary resuscitation: how useful? Attitudes and knowledge of an elderly population. Arch Intern Med. 1992;152(3):578-582.
2. Schonwetter RS, Walker RM, Kramer DR, Robinson BE. Resuscitation decision making in the elderly: the value of outcome data. J Gen Intern Med. 1993;8(6):295-300.

3. Mead GE, Turnbull CJ. Cardiopulmonary resuscitation in the elderly: patients' and relatives' views. J Med Ethics. 1995;21(1):39-44.

4. Frank C, Heyland DK, Chen B, Farquhar D, Myers K, Iwaasa K. Determining resuscitation preferences of elderly inpatients: a review of the literature. CMAJ. 2003;169(8):795-799.

5. Miller DL, Gorbien MJ, Simbartl LA, Jahnigen DW. Factors influencing physicians in recommending in-hospital cardiopulmonary resuscitation. Arch Intern Med. 1993;153(17):1999-2003.

6. Murphy DJ, Burrows D, Santilli S, et al. The influence of the probability of survival on patients' preferences regarding cardiopulmonary resuscitation. N Engl J Med. 1994;330(8):545-549.

7. van Gijn MS, Frijns D, van de Glind EM, B CvM, Hamaker ME. The chance of survival and the functional outcome after in-hospital cardiopulmonary resuscitation in older people: a systematic review. Age Ageing 2014;43(4):456-463.

8. Galea S, Blaney S, Nandi A, et al. Explaining racial disparities in incidence of and survival from out-of-hospital cardiac arrest. Am $J$ Epidemiol 2007; 166(5):534-543.

9. Ehlenbach WJ, Barnato AE, Curtis JR, et al. Epidemiologic study of inhospital cardiopulmonary resuscitation in the elderly. $N$ Engl $J$ Med 2009;361(1):22-31.

10. Tokudome $\mathbf{S}$, Hashimoto $\mathbf{S}$, Igata $\mathbf{A}$. Life expectancy and healthy life expectancy of Japan: the fastest graying society in the world. BMC Res Notes 2016;9(1):482.

11. Sasaki A, Hiraoka E, Homma Y, et al. Association of code status discussion with invasive procedures among advanced-stage cancer and noncancer patients. Int $J$ Gen Med 2017;10:207-214.

12. Tanaka S, Seto K, Kawakami $\mathbf{K}$. Pharmacoepidemiology in Japan: medical databases and research achievements. J Pharm Health Care Sci 2015;1:16.

13. Sumitani $\mathbf{M}$, Uchida $\mathbf{K}$, Yasunaga $\mathbf{H}$, et al. Prevalence of malignant hyperthermia and relationship with anesthetics in Japan: data from the diagnosis procedure combination database. Anesthesiology 2011;114(1):84-90

14. Isogai T, Yasunaga $\mathbf{H}$, Matsui $\mathbf{H}$, et al. Effect of weekend admission for acute myocardial infarction on in-hospital mortality: a retrospective cohort study. Int $J$ Cardiol 2015; 179:315-320.

15. Shigematsu $\mathbf{K}$, Nakano $\mathbf{H}$, Watanabe $\mathbf{Y}$. The eye response test alone is sufficient to predict stroke outcome-reintroduction of Japan Coma Scale: a cohort study. BMJ Open. 2013;3(4).

16. Charlson ME, Pompei P, Ales KL, MacKenzie CR. A new method of classifying prognostic comorbidity in longitudinal studies: development and validation. $J$ Chronic Dis 1987;40(5):373-383.

17. Larkin GL, Copes WS, Nathanson BH, Kaye W. Pre-resuscitation factors associated with mortality in 49,130 cases of in-hospital cardiac arrest: a report from the National Registry for Cardiopulmonary Resuscitation. Resuscitation 2010;81(3):302-311.

18. Nadkarni VM, Larkin GL, Peberdy MA, et al. First documented rhythm and clinical outcome from in-hospital cardiac arrest among children and adults. JAMA 2006;295(1):50-57.

19. Peberdy MA, Kaye W, Ornato JP, et al. Cardiopulmonary resuscitation of adults in the hospital: a report of 14720 cardiac arrests from the National Registry of Cardiopulmonary Resuscitation. Resuscitation 2003;58(3):297-308.

20. Peberdy MA, Ornato JP, Larkin GL, et al. Survival from in-hospital cardiac arrest during nights and weekends. JAMA 2008;299(7):785-792.

21. Koike T, Nakagawa M, Shimozawa $\mathbf{N}$, et al. The implementation of rapid response system in Japanese hospitals: its obstacles and possible solutions. J Jap Assoc Acute Med 2017;28(6):219-229 (in Japanese).

22. Yamana H, Moriwaki M, Horiguchi H, Kodan M, Fushimi K, Yasunaga $\mathbf{H}$. Validity of diagnoses, procedures, and laboratory data in Japanese administrative data. $J$ Epidemiol 2017;27(10):476-482. 